\title{
Childhood adiposity and adolescent sex steroids in the Exploring Perinatal Outcomes among Children study
}

\author{
Catherine Kim ${ }^{1}$ (D) | Kylie K. Harrall ${ }^{2}$ | Deborah H. Glueck ${ }^{2,3}$ | Daniel Shumer ${ }^{4}$ | \\ Dana Dabelea ${ }^{2,3,5}$
}

\author{
${ }^{1}$ Departments of Medicine, Obstetrics \& \\ Gynecology, and Epidemiology, University of \\ Michigan, Ann Arbor, Michigan \\ ${ }^{2}$ Lifecourse Epidemiology of Adiposity \\ and Diabetes (LEAD) Center, University of \\ Colorado Denver, Aurora, Colorado \\ ${ }^{3}$ Department of Pediatrics, University \\ of Colorado School of Medicine, Aurora, \\ Colorado \\ ${ }^{4}$ Department of Epidemiology, University of \\ Colorado, Aurora, Colorado \\ ${ }^{5}$ Department of Pediatrics, University of \\ Michigan, Ann Arbor, Michigan

\section{Correspondences} \\ Catherine Kim, Departments of Medicine, \\ Obstetrics \& Gynecology, and Epidemiology, \\ University of Michigan, Ann Arbor, MI. \\ Email: cathkim@umich.edu \\ Funding information \\ National Institute of General Medical \\ Sciences, Grant/Award Number: \\ R01GM121081; National Institutes \\ of Health, Grant/Award Number: \\ 5UG3OD023248, M01 RR00069, \\ R01DK076648 and RO1 DK068001307
}

\begin{abstract}
Objective: It is unclear how childhood adipose tissue deposition influences sex hormone profiles in later adolescence.

Design: Prospective cohort study.

Participants: Children $(n=418)$ with a mean age of $10.5(1.5)$ years at visit 1 and 16.7 (1.2) at visit 2 in the Exploring Perinatal Outcomes among Children (EPOCH) Study.

Measurements: We used reverse-scale Cox proportional hazard models to assess associations between pubertal dehydroepiandrosterone (DHEA), testosterone (T), and oestradiol (E2) and childhood-to-puberty rate of change in visceral (VAT) and subcutaneous adipose tissue (SAT). Models stratified by sex and adjusted for childhood adiposity, maternal factors, birthweight and pubertal onset, and then further adjusted for insulin, luteinizing hormone (LH), leptin and hepatic fat fraction.

Results: Among boys, more rapid accumulation of either VAT or SAT was associated with lower testosterone at visit 2 (HR 0.86, and .96, respectively, both $P<.0001$ ), independently of race/ethnicity, LH, leptin and hepatic fat fraction. Among boys, more childhood VAT was associated with lower testosterone in adolescence (HR 0.98, $P=.003)$, but this association did not persist after adjustment for leptin or insulin. No associations were observed between either fat measure and oestradiol or DHEA in boys. In girls, no associations between childhood fat or fat accumulation and sex hormones were observed.

Conclusions: More rapid accumulation of fat is associated with lower testosterone in boys. These associations suggest that fat growth influences androgen profiles in adolescent boys. Since fat accumulation is a modifiable risk factor, the study results provide a possible intervention target and time period for improving adult health.

\section{KEYWORDS}

adipose tissue, adolescent, testosterone, visceral adiposity
\end{abstract}

\section{1 | INTRODUCTION}

The pubertal transition represents a period of dramatic alterations in body composition and sex hormone profiles. Previous reports in girls ${ }^{1-4}$ suggest that obesity is associated with earlier onset of puberty and hyperandrogenemia, whereas the opposite may be true in boys. ${ }^{5,6}$ As most of these reports were cross-sectional, it is unclear whether the speed of fat accumulation or baseline body fat influenced the onset of puberty, and to what extent sex steroid hormones as opposed to other markers of puberty were altered. How growth 
in specific adipose tissue depots, particularly visceral adipose tissue (VAT) and subcutaneous adipose tissue (SAT), are associated with sex profiles in adolescence is also not established. Finally, adipose tissue production of leptin may stimulate gonadotropin secretion and luteinizing hormone (LH) release as well as insulin resistance, but whether the associations between fat accumulation and sex hormones occur after accounting for luteinizing hormone (LH), fasting insulin, hepatic fat deposition and leptin has not been studied.

The Exploring Perinatal Outcomes in Children (EPOCH) study is a longitudinal cohort study of youth in Colorado.' Approximately 418 children had magnetic resonance imaging (MRI) assessed VAT and SAT during prepuberty and early puberty, followed by repeated adiposity and sex hormone measures six years later. In this report, we examined the associations between baseline VAT and SAT in childhood, and growth rate in VAT and SAT with sex hormone levels later in adolescence. We also examined whether these associations changed after adjusting for potential mediators including $\mathrm{LH}$, fasting insulin, hepatic fat and fasting leptin concentrations. Based on previous studies in children, we hypothesized that greater VAT and VAT growth would be associated with higher testosterone levels in girls at the follow-up visit, ${ }^{1-4}$ whereas greater VAT and VAT growth would be associated with lower testosterone levels in boys. ${ }^{5,6}$ Based on studies in adult women, ${ }^{8}$ we also hypothesized that greater SAT would be associated with higher oestradiol levels in girls at the follow-up visit.

\section{2 | MATERIALS AND METHODS}

\section{1 | Participants}

The design, methods and baseline characteristics of EPOCH participants have been previously described. ${ }^{7} \mathrm{EPOCH}$ is an observational prospective study that recruited healthy 6- to 13-year-old children who were offspring of singleton pregnancies, born at a single hospital in Denver between 1992 and 2002, whose biological mothers were members of Kaiser Permanente of Colorado (KPCO). The study population was sampled to reflect similar racial and ethnic distributions of Colorado. The EPOCH study included an oversampling of offspring of mothers who had experienced gestational diabetes in pregnancy, and average $\mathrm{BMI}$ in $\mathrm{EPOCH}$ is slightly higher than the national standard. ${ }^{9}$ Children and their mothers were invited to participate in two research visits at average ages of $10.5(\mathrm{SD}=1.5)$ and 16.7 (SD = 1.2). While a total of 604 children attended the 1 st research visit, this analysis focused on the 418 children who attended both visits. All participants provided informed consent, and youths provided written assent. The study was approved by the Colorado Multiple Institutional Review Board.

\section{2 | Measures of adiposity}

Childhood height and weight were measured in light clothing and without shoes. Weight was measured to the nearest $0.1 \mathrm{~kg}$ using an electronic scale. Height was measured to the nearest $0.1 \mathrm{~cm}$ using a portable stadiometer. BMI was calculated as $\mathrm{kg} / \mathrm{m}^{2}$. Waist circumference was measured according to the National Health and Nutrition Examination Survey protocol as previously described. ${ }^{10}$ At both visits, MRI of the abdominal region was used to quantify visceral adipose tissue (VAT) and subcutaneous adipose tissue (SAT) with a 3 T HDx Imager (General Electric) by a trained technician. Each study participant was placed supine and a series of T1-weighted coronal images were taken to locate the L4/L5 plane. One axial, $10 \mathrm{~mm}$, T1-weighted image, at the umbilicus or L4/L5 vertebrae, was analysed to determine SAT and VAT content. Images were analysed by a single reader, blinded to exposure status. With measures of VAT and SAT, we generated VAT/SAT ratios. Previous reports in the EPOCH cohort have examined the correlation between BMI and specific markers of visceral adiposity. BMI has high correlation with SAT in boys (Pearson's $r=.94$ ) and girls $(r=.91)$, but the correlation with VAT is moderate $(r=.65$ in boys, $r=.68$ in girls); correlation between visceral fat mass and waist circumference was similar. ${ }^{10}$ Hepatic imaging was performed at the 2nd research visit using a magnitude based, 6-echo, spoiled gradient-recalled echo sequence. Hepatic fat fraction (HFF) was calculated from the mean pixel signal intensity data, for each echo acquisition using an open source Osirix algorithm. This fraction was then multiplied by 100 such that a value of 1 is equivalent to $1 \%$ HFF.

\section{3 | Dehydroepiandrosterone, testosterone, oestradiol measurements}

A fasting blood draw after an overnight fast occurred for all consenting children at the second visit. Sera were refrigerated and analysed within 24 hours of collection. All laboratory measurements were performed at the Colorado Clinical Translational Science Institute Core Laboratories. Serum oestradiol was measured by using a Beckman Coulter chemiluminescent with a limit of detection of $10.0 \mathrm{pg} / \mathrm{mL}$. Serum total testosterone was measured by using a Beckman Coulter 1-step competitive with a limit of detection of $17 \mathrm{ng} / \mathrm{dL}$. Serum DHEA was measured by using a Beckman Coulter chemiluminescent with a limit of detection of $2 \mu \mathrm{g} / \mathrm{dL}$.

\section{4 | Covariate measurements}

The KPCO Perinatal database, an electronic database linking the neonatal and perinatal medical record, was used to collect birthweight. Race/ethnicity was self-reported using 2000 US Census-based questions and categorized as Hispanic (any race), non-Hispanic white, non-Hispanic African American and non-Hispanic other. Pubertal development was self-assessed using a diagrammatic representation of Tanner staging adapted from Marshall and Tanner; sexual maturation self-assessment was recently shown to be in excellent agreement with physician-assessed Tanner stage, ${ }^{11}$ although lack of direct testicular measurement could potentially over-estimate pubertal stage. For the purpose of the analysis, youth were categorized as prepubertal (Tanner $<2$ ) and pubertal (Tanner $2-5$ ), and as 
TABLE 1 Participant characteristics in EPOCH. Means (standard deviations [SD]) or $\mathrm{n}$ (per cent) shown

\begin{tabular}{|c|c|c|c|c|}
\hline & \multicolumn{2}{|c|}{ Boys $(\mathrm{N}=209)$} & \multicolumn{2}{|c|}{ Girls (N = 209) } \\
\hline & Visit 1 & Visit 2 & Visit 1 & Visit 2 \\
\hline Age (y) & $10.5(1.5)$ & $16.6(1.2)$ & $10.4(1.5)$ & $16.7(1.2)$ \\
\hline \multicolumn{5}{|l|}{ Stage of puberty (n, \%) } \\
\hline Tanner 1 & $113(54 \%)$ & 0 & $74(35 \%)$ & 0 \\
\hline Tanner 2 & 65 (31\%) & $3(1 \%)$ & 77 (37\%) & 0 \\
\hline Tanner 3 & $24(12 \%)$ & $5(2 \%)$ & $38(18 \%)$ & $16(8 \%)$ \\
\hline Tanner 4 & $6(3 \%)$ & $73(35 \%)$ & $19(9 \%)$ & 93 (45\%) \\
\hline Tanner 5 & 0 & $126(61 \%)$ & $1(0.5 \%)$ & $100(48 \%)$ \\
\hline \multicolumn{5}{|l|}{ Race/ethnicity (n, \%) } \\
\hline Non-Hispanic White & $101(48 \%)$ & & $112(54 \%)$ & \\
\hline Hispanic & 81 (39\%) & & $68(33 \%)$ & \\
\hline African American & $15(7 \%)$ & & $18(9 \%)$ & \\
\hline Other & $12(6 \%)$ & & $11(5 \%)$ & \\
\hline Birthweight (lbs) & $7.9(4.7)$ & & $7.6(4.5)$ & \\
\hline $\begin{array}{l}\text { Maternal education, high school or } \\
\text { less (n, \%) }\end{array}$ & $35(17 \%)$ & & $39(19 \%)$ & \\
\hline Maternal income $<\$ 50000(\mathrm{n}, \%)$ & $98(47 \%)$ & & 89 (43\%) & \\
\hline Body mass index $\left(\mathrm{kg} / \mathrm{m}^{2}\right)$ & $19.1(4.7)$ & $23.7(5.5)$ & $18.5(4.2)$ & $23.6(5.7)$ \\
\hline Fasting insulin (IU/L) & & $16.3(11.3)$ & & 17.5 (11.4) \\
\hline Luteinizing hormone (IU/L) & & $4.2(3.9)$ & & $10.7(31.3)$ \\
\hline Leptin (ng/mL) & & $6.6(8.2)$ & & $19.6(15.4)$ \\
\hline Hepatic fat fraction (\%) & & $2.7(3.7)$ & & $2.2(2.2)$ \\
\hline Visceral adiposity (VAT) $\left(\mathrm{cm}^{2}\right)$ & $22(15)$ & $32(22)$ & $22(15)$ & $34(22)$ \\
\hline Subcutaneous adiposity (SAT) $\left(\mathrm{cm}^{2}\right)$ & $116(113)$ & $172(154)$ & $119(98)$ & $232(144)$ \\
\hline VAT/SAT & $0.26(0.14)$ & $0.24(0.13)$ & $0.22(0.10)$ & $0.16(0.06)$ \\
\hline Oestradiol (pg/mL) & & $27.0(12.5)$ & & $\begin{array}{l}86.3 \\
(86.6)\end{array}$ \\
\hline Testosterone (ng/dL) & & $\begin{array}{l}489.5 \\
(146.8)\end{array}$ & & $41.1(16.0)$ \\
\hline DHEA (ng/dL) & & $\begin{array}{l}226.8 \\
(107.7)\end{array}$ & & $\begin{array}{c}174.4 \\
(83.1)\end{array}$ \\
\hline
\end{tabular}

white or nonwhite. Maternal level of education (high school or less vs more than high school) and total household income $(<\$ 50000$ vs more than $\$ 50$ 000) were self-reported at the study visit. Fasting insulin was measured by a radioimmunoassay method. Plasma leptin concentration was measured by using a Millipore radioimmunoassay with a sensitivity of $0.5 \mathrm{ng} / \mathrm{mL}$. Serum LH was determined by using a Beckman Coulter chemiluminescent assay with a sensitivity of $0.12 \mathrm{mIU} / \mathrm{mL}$.

\section{5 | Statistical analysis}

Girls and boys were examined separately. Baseline characteristics were described using sample size and percentages for categorical variables and means and standard deviations for numeric variables (Table 1). When considering sex steroid profiles, given the young age of study participants, we needed to consider the substantial number of values that fell below the level of detection. ${ }^{9-12}$ Many authors choose to replace values below the limit of detection with randomly sampled values, or with a value halfway between zero and the detection limit. These approaches have been critiqued for introducing bias or reducing statistical power. ${ }^{12-15}$ We chose instead to employ the reverse-scale Cox proportional hazards approach, ${ }^{16}$ an unbiased approach which maintains power by including both values above and below the limit of detection. The approach does not rely on either estimation or imputation. ${ }^{16}$

In classical survival analysis, the data are right-censored. By contrast, sex hormone data are left-censored at the level of detection. It is important to realize that data below the level of detection still conveys information. Intuitively, if a person has a sex hormone level below the limit of detection, the level must be very low. This leaves the analyst with a thorny problem. How do we apply methods for right-censored data to left-censored data? 


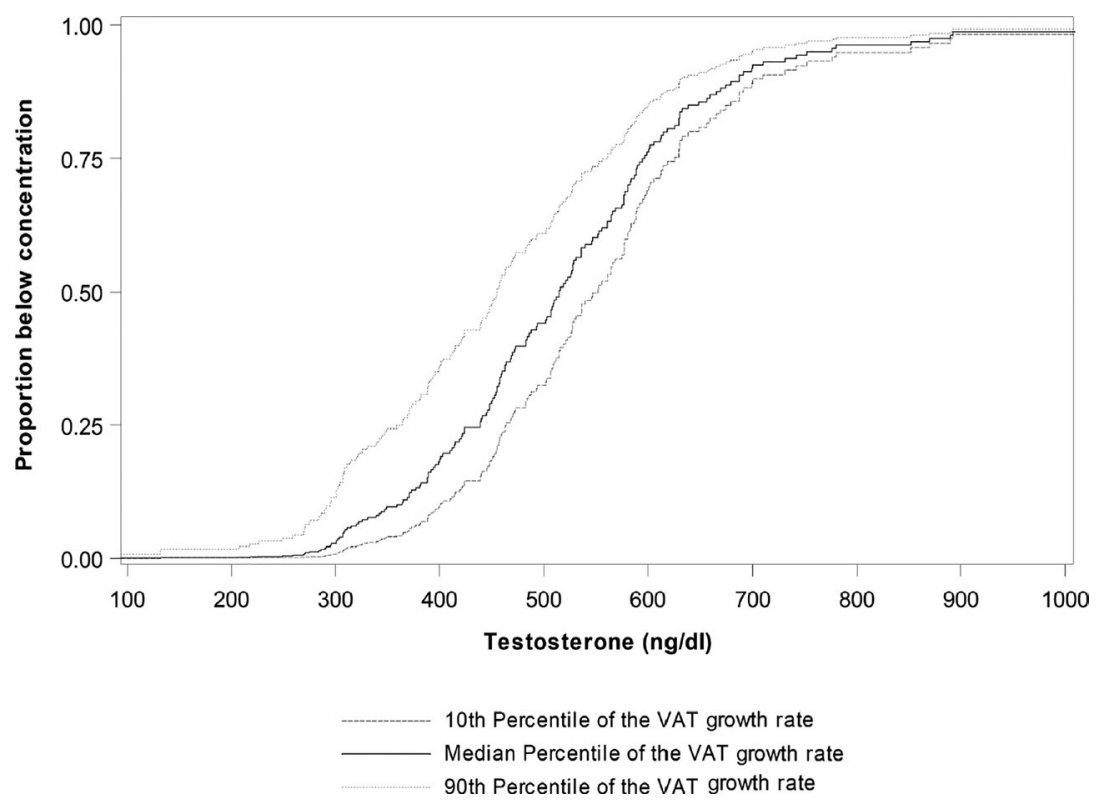

FIGURE 1 Proportion of adolescent boys with testosterone levels below the level of detection at their follow-up visit, by percentiles of the VAT growth rate
To apply classical survival methods to sex hormone data, the scale of the data must be reversed, through a transformation. The scale of each sex hormone is reversed by subtracting observed sex hormone levels from the maximum observed value for that sex hormone in the data set. This effectively converts left-censored to rightcensored data. The resulting model predicts the risk of sex steroid levels above the limit of detection. Thus, a hazard ratio less than 1 indicates a greater risk of having undetectable sex steroid levels. The approach allows the analyst to describe the percentage of the population below any sex steroid hormone level of interest, as shown in Figure 1.

We fit nine separate reverse-scale Cox proportional hazard models. Each model had a different hormone outcome (DHEA, testosterone or oestradiol) and a different measure of adiposity as a predictor (VAT, SAT, or VAT/SAT). For the best fitting models, we assessed the validity of the proportional hazard assumption, and reported model generated hazard ratios, parameter estimates, $95 \%$ confidence intervals and graphics.

The base model included the specific fat measure at visit 1 , growth rate in adiposity between the two visits, and the following covariates: maternal education and income, birthweight and pubertal stage at visit 1 . Growth rate in adiposity was computed as the difference between the adiposity measure at visit 2 and the measure at visit 1 , divided by the difference in age between visit 2 and age at visit 1 . Subsequent models further adjusted for visit $2 \mathrm{LH}$, fasting insulin, leptin and hepatic fat to determine whether addition of these potential mediators changed the association. We also evaluated interactions between race/ethnicity with the initial fat measure and growth rate in that specific fat measure; if no interactions were detected, models were adjusted only for the main effect of race/ethnicity. Analyses were performed using the Statistical Analysis Software (SAS) version 9.4 (SAS Institute), and all tests were two-sided with statistical significance set at $P<.05$.

\section{3 | RESULTS}

Table 1 shows participant characteristics by sex. At the first visit, both boys and girls were between 6 and 14 years old (on average $10.5 \pm 1.5$ years), and at the second visit, both boys and girls were between 12 and 20 years old (on average $16.7 \pm 1.2$ years). At the first visit, approximately half of the boys were prepubertal compared to about $1 / 3$ of the girls; by visit 2 , all of the children had reached the onset of puberty. One boy was Tanner stage 2 at $<11.5$ years of age and one girl was Tanner stage 2 at $<10.5$ years of age, and conversely, no girls were Tanner stage 1 at 12 years of age and no boys were Tanner stage 1 at 14 years of age. About half were non-Hispanic white. Although most mothers had more than a high school education, almost half of the population had an annual income less than $\$ 50000$ per year. Table 1 also shows average adipose tissue areas and sex steroid levels. While DHEA concentrations were consistently above the level of detection, a substantial proportion of children had oestradiol and testosterone concentrations below the limit of detection. At visit 2, approximately $12 \%$ of girls and $1 \%$ of boys had undetectable testosterone levels, and $7 \%$ of girls and $6 \%$ of boys had undetectable oestradiol levels.

Among boys, Table 2 shows the association between change in adipose tissue depots between visit 1 and visit 2, as well as baseline adipose tissue, with the risk of undetectable hormones at visit 2 . Even after adjustment for baseline VAT and SAT, more rapid growth in VAT and more rapid growth in SAT were both associated with higher risk of undetectable testosterone at follow-up (due to reverse-scale Cox proportional hazards modelling, an $\mathrm{HR}<1$ indicates greater risk). For every $1 \mathrm{~mm}^{2}$ increase in VAT, there was a $14 \%$ increase in the risk of testosterone values below the limit of detection (HR: 0.86, $P<.0001$ ). For every $1 \mathrm{~mm}^{2}$ increase in SAT, there was a $4 \%$ increase in the risk of testosterone values below the limit of detection (HR: 0.96, $P<.0001)$.

Figure 1 shows the estimated proportions of the adolescent male population below a specific testosterone level. Different lines 
TABLE 2 Association between change in adiposity between visit 1 and visit 2 (predictor) with circulating sex steroid hormones at visit 2 (outcome). Hazard ratios and $95 \%$ confidence intervals shown (HR, 95\% Cl); hazard ratios < 1 indicate greater risk for undetectable sex steroid concentrations at visit 2

\begin{tabular}{|c|c|c|c|}
\hline \multirow{3}{*}{ Boys-VAT models } & \multirow{2}{*}{$\frac{\text { Testosterone }}{\text { HR }(95 \% \mathrm{CI})}$} & \multirow{2}{*}{$\frac{\text { Oestradiol }}{\operatorname{HR}(95 \% \mathrm{Cl})}$} & \multirow{2}{*}{$\frac{\text { DHEA }}{\text { HR }(95 \% \mathrm{CI}}$} \\
\hline & & & \\
\hline & & & \\
\hline \multicolumn{4}{|c|}{ Adjusted for birthweight, maternal education, incom } \\
\hline VAT at visit 1 & $0.98(0.003)$ & $1.00(0.60)$ & $1.00(0.99)$ \\
\hline Change in VAT & $0.86(<0.0001)$ & $0.99(0.61)$ & $0.97(0.30)$ \\
\hline \multicolumn{4}{|l|}{ Boys-SAT models } \\
\hline \multicolumn{4}{|c|}{ Adjusted for birthweight, maternal education, income, visit 1 pubertal stage } \\
\hline SAT at visit 1 & $1.00(0.07)$ & $1.00(0.40)$ & $1.00(0.57)$ \\
\hline Change in SAT & $0.96(<0.0001)$ & $1.00(0.28)$ & $0.99(0.17)$ \\
\hline \multicolumn{4}{|l|}{ Boys-VAT/SAT models } \\
\hline \multicolumn{4}{|c|}{ Adjusted for birthweight, maternal education, income, visit 1 pubertal stage } \\
\hline VAT/SAT at visit 1 & $4.04(0.028)$ & $0.55(0.36)$ & $1.03(0.97)$ \\
\hline Change in VAT/SAT & $0.61(0.32)$ & $0.11(0.61)$ & $0.11(0.59)$ \\
\hline \multicolumn{4}{|l|}{ Girls-VAT models } \\
\hline \multicolumn{4}{|c|}{ Adjusted for birthweight, maternal education, income, visit 1 pubertal stage } \\
\hline VAT at visit 1 & $0.99(0.13)$ & $1.00(0.93)$ & $1.00(0.63)$ \\
\hline Change in VAT & $1.01(0.82)$ & $1.00(0.95)$ & $1.00(0.95)$ \\
\hline \multicolumn{4}{|l|}{ Girls-SAT models } \\
\hline \multicolumn{4}{|c|}{ Adjusted for birthweight, maternal education, income, visit 1 pubertal stage } \\
\hline SAT at visit 1 & $1.00(0.76)$ & $1.00(0.50)$ & $1.00(0.26)$ \\
\hline Change in SAT & $1.00(0.93)$ & $1.00(0.72)$ & $1.00(0.55)$ \\
\hline \multicolumn{4}{|l|}{ Girls-VAT/SAT models } \\
\hline \multicolumn{4}{|c|}{ Adjusted for birthweight, maternal education, income, visit 1 pubertal stage } \\
\hline VAT/SAT at visit 1 & $0.20(0.24)$ & $0.61(0.69)$ & $2.10(0.56)$ \\
\hline Change in VAT/SAT & $0.02(0.70)$ & $4.01(0.88)$ & $5420(0.39)$ \\
\hline
\end{tabular}

The bold values are values that are significant at $P<0.05$. show the different curves by percentile of VAT growth rate. The curve for the 10th percentile in VAT growth rate lies at the bottom, and the curve for the 90th percentile lies at the top. The estimates are drawn from reverse Cox proportional hazard models. The VAT growth rate is treated as a continuous predictor, so as the rate of growth in VAT increases, the proportion of the population below a specific testosterone value increases. Estimates can be obtained for any percentile desired: only the 10th, 50th and 90th are shown here for clarity.

Higher levels of VAT at baseline were also associated with a $2 \%$ increase in the risk of undetectable testosterone at visit 2 (HR: 0.98, $P<$.003). However, higher concentrations of VAT/SAT at baseline were associated with a lower risk of undetectable testosterone at future visits (HR: 4.04, $P=.028$ ), suggesting that boys with relative large amounts of visceral fat relative to subcutaneous fat prior to the pubertal transition were more likely to have detectable testosterone levels at follow-up. These patterns did not change with adjustment for race/ethnicity (results not shown). Among girls, no fat depots or change in fat depots were associated with future sex steroid levels (Table 2).
Among boys, the associations between growth rate of VAT and growth rate of SAT with testosterone persisted after adjustment for other variables, including insulin, LH, leptin and hepatic fat (Table 3), suggesting that these factors did not account for the association between growth of fat with future low testosterone. The association between baseline VAT with future testosterone was attenuated in models including fasting insulin and models including leptin, suggesting that these variables either confounded or mediated the relationship between baseline VAT and future testosterone. Similarly, the association between baseline VAT/SAT with future testosterone was also attenuated by fasting insulin and leptin, suggesting that these variables either confounded or mediated the relationship between baseline VAT/SAT and future testosterone. These associations also persisted after adjustment for race/ethnicity (results not shown).

\section{4 | DISCUSSION}

In a cohort of children who had not yet completed their pubertal transition, we found that, among boys, more rapid growth in 


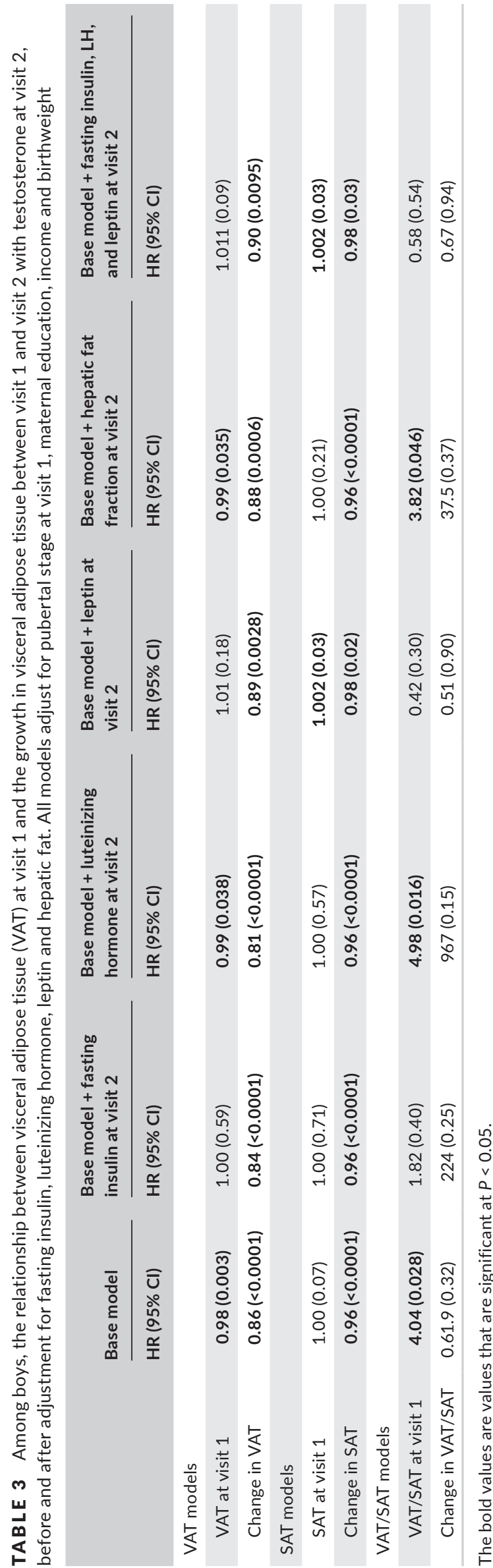

fat depots predicted greater risk for low testosterone concentrations roughly 6 years later. These associations were independent of other factors known to affect testosterone production, including LH, insulin, leptin and hepatic fat. These associations were also independent of baseline quantities of fat. These findings suggest that the speed of fat accumulation, a modifiable exposure, is an important determinant of future androgen profiles in boys. We did not observe relationships between fat depot and hormone profiles in girls, suggesting that neither VAT nor SAT are independent determinants of sex steroids in healthy girls without precocious puberty.

Although adolescence represents a time of relatively rapid accumulations in adipose tissue and changes in sex steroids, few reports have examined whether the associations observed in adults are also present in healthy children as they accumulate fat and transition through puberty.

It has long been recognized that greater body weight and body mass index in adult men is inversely related to testosterone concentrations, particularly higher fat mass. ${ }^{17}$ Fewer studies have been able to examine whether changes in fat mass over time lead to changes in sex steroids, although these reports also suggest that the loss or accumulation of fat can influence sex steroids. In one secondary analysis of a randomized trial of weight loss, we found that reductions in VAT and SAT resulted in increases in testosterone among overweight adult men. ${ }^{18}$ In two other observational cohorts, men with the greatest weight gains had the greatest declines in testosterone over time. ${ }^{19,20}$ Prepubertal obese boys tend to have higher testosterone than nonobese boys ${ }^{21,22}$ as well as earlier timing of puberty, ${ }^{23}$ but obese pubertal boys have lower testosterone levels than nonobese pubertal boys. ${ }^{24,25}$

Our findings build upon this literature in several respects. First, we note that the rapidity of accumulation of fat is important for boys, with more rapid accumulations increasing risk of low testosterone in later adolescence. This represents a potentially modifiable exposure for children who are obese. Visceral adipose tissue growth, as opposed to growth of other tissues, is difficult to assess using metrics that are easily accessible to the practicing clinician. However, lower energy intake from fat and higher levels of aerobic physical activity predict fat deposition in adolescents. ${ }^{26,27}$ Thus, for the practicing paediatrician, the emphasis on these healthy behaviours with the intention of limiting harmful fat accumulation can, in turn, influence sex hormone profiles. Preventing rapid gains in fat could ameliorate downstream effects on sex hormones. This implies that slowing the rate of such fat accumulation will affect androgen levels in adolescence, which in turn may impact future cardiovascular risk. ${ }^{28}$

Second, we were able to quantify fat depots precisely through the use of MRI as opposed to anthropometric measures, which may not represent adiposity in specific compartments. In contrast, we did not find associations between fat mass and hormone levels in girls. Previous studies have noted that girls have testosterone levels directly correlated with degree of obesity, $2,3,29$ particularly VAT. ${ }^{29}$ Our results may have differed from these 
previous reports for several reasons. We conducted a longitudinal examination of the association between fat and future testosterone, and it is possible that fat mass in girls has relatively little effect, compared with other factors that are also rapidly changing during the pubertal transition. ${ }^{3}$ These factors include $\mathrm{LH}$ and fasting insulin, which are related to testosterone levels, even after adjustment for adiposity and age. ${ }^{3}$ In addition, we examined the relationship between specific fat depots rather than overall body mass, and it is possible that the associations previously observed between body mass and hormone levels reflect other fat compartments than VAT and SAT.

Finally, studies have focused upon the relationship between obesity and puberty timing, rather than sex steroid levels or specific fat measures per se. Puberty is a complex transition of which sex hormones are only a single dimension, and thus it is possible that a stronger relationship exists between obesity and puberty than between VAT, SAT and future sex steroid concentrations in girls.

Among boys, the associations between rate of fat mass accumulation and low testosterone later in adolescence remained significant after controlling for several potential mediators or confounders of the relationship between fat and testosterone, namely insulin, ${ }^{30}$ leptin, ${ }^{31} \mathrm{LH}^{31}$ and hepatic fat fraction. ${ }^{32}$ In contrast, the relationships of baseline VAT and baseline SAT with future testosterone were no longer significant after adjustment for these factors, consistent with prior studies suggesting a potential mediating role of these factors. This suggests that rapid accumulations in fat affect sex steroid production through other pathways. Although speculative, such pathways could include gut hormones such as ghrelin, which may have inhibitory effects upon the reproductive axis. ${ }^{33}$ Other potential pathways include other adipokines that may affect sex steroid production through direct effects on the gonads or through hypothalamic-pituitary pathways. ${ }^{33}$ Although the majority of circulating androgens in boys originate in the testes, small amounts of the adrenal androgen dehydroepiandrosterone are converted to androstenedione and then to testosterone. The lack of association between VAT and SAT with DHEA suggests that these particular fat depots did not have a large impact on adrenal androgen production. However, it is possible that adrenal androgen production may have influenced deposition of fat and possibly gonadal testosterone production. ${ }^{34}$ Finally, despite the fact that the associations between fat mass accumulation and lower testosterone persist after controlling for $\mathrm{LH}$, the interpretation of this is complicated by the fact that testosterone and LH function in a feedback loop. Normal LH concentrations in a male with hypogonadism may actually signify an LH level that is inappropriately low. Therefore, the finding that fat accumulation and low testosterone are associated while controlling for LH may inappropriately minimize the role of obesity in affecting the hypothalamic-pituitary-testicular axis and its healthy functioning.

The strengths of this report include a relatively large, diverse longitudinal cohort which characterized adiposity using MRI at 2 points in time along with sex hormone measures and possible mediators. We also used a novel and powerful method of analysis ${ }^{16}$ that allowed us to meaningfully interpret sex hormone levels below the level of detection, while avoiding potential bias due to imputation or extrapolation of data. However, there are several limitations. We examined multiple sex steroids, and we performed multiple comparisons. Thus, some of the observed associations may have been due to chance and need to be replicated. The optimal method of estimating bioavailable testosterone and oestradiol in lieu of direct measurements is controversial due to possible changes in binding affinity with age and population characteristics. Thus, we did not measure free fractions of sex steroids and sex hormone binding globulin, and it is possible that use of these measures might yield a different pattern of results. We considered this issue carefully in constructing the analysis plan, which rests on the probability of absence of detectable testosterone. Since total testosterone exists in equilibrium with free testosterone, the lack of detectable total testosterone likely indicates low levels of free testosterone, regardless of SHBG levels. If we had examined level of testosterone as the outcome with a conventional survival analysis, we agree the lack of SHBG would be a significant limitation. We did adjust for hepatic fat fraction, which is an indicator of fatty liver and correlated with sex hormone binding globulin, and adjustment did not alter the pattern of results. Body composition may have bidirectional associations with sex hormones, ${ }^{18}$ and it is possible that changes in sex hormones influenced body fat deposition. To examine whether this is the case, we would need to examine whether sex hormone changes predicted VAT and SAT at visit 2, after adjustment for baseline VAT and SAT, which we plan to examine in the future.

We conclude that the rate of fat accumulation in children may influence their hormone profiles in later puberty, and specifically that more rapid fat accumulation in boys appears to be associated with subsequent risk of low testosterone. These associations do not seem to occur via insulin levels, hepatic fat, LH or leptin. Future studies should explore the potential role of other adipokines or gut peptides upon sex steroid production, and whether sex steroid profiles in children alter risk of future adiposity and sex hormone disorders in adults. In order to determine whether the impact of VAT upon sex steroid profile persists when participants have completed puberty, another assessment of the cohort is planned when participants are approximately 22 years of age. Until that assessment, it is not known whether the impact of obesity upon sex steroid profiles is limited to the late-pubertal stage.

\section{ACKNOWLEDGEMENTS}

The Research Group gratefully acknowledges the commitment and dedication of the participants of the EPOCH study. This work was funded by grants 5UG3OD023248, R01DK076648 and R01GM121081 from the National Institutes of Health. The EPOCH study was supported by General Clinical Research 308 Centers Program (RO1 DK068001307 to DD and M01 RR00069). The study sponsors had no role in the study. 


\section{CONFLICT OF INTEREST}

The authors have no conflict of interest. This work was presented in part at the 2019 Scientific Sessions of the American Diabetes Association.

\section{AUTHOR CONTRIBUTIONS}

CK wrote the manuscript and is the guarantor of the manuscript. $\mathrm{KKH}$ and DHG performed the analysis and revised and edited the manuscript. DS reviewed and edited the document and contributed to discussion of the manuscript. DD directed the EPOCH study, obtained research data, reviewed/edited and contributed to discussion of the manuscript.

\section{DATA AVAILABILITY}

The data that support the findings of this study are available from the corresponding author upon reasonable request.

\section{ORCID}

Catherine Kim (iD https://orcid.org/0000-0001-9237-0532

\section{REFERENCES}

1. Ibanez L, Ong K, de Zegher F, Marcos M, del Rio L, Dunger D. Fat distribution in non-obese girls with and without precocious pubarche: central adiposity related to insulinaemia and androgenaemia from puberty to postmenarche. Clin Endocrinol. 2003;58:372-379.

2. McCartney CR, Blank SK, Prendergast KA, et al. Obesity and sex steroid changes across puberty: evidence for marked hyperandrogenemia in pre- and early pubertal obese girls. J Clin Endocrinol Metab. 2007;92:430-436.

3. Knudsen KL, Blank SK, Burt Solorzano C, et al. Hyperandrogenemia in obese peripubertal girls: correlates and potential etiological determinants. Obesity. 2010;18(11):2118-2124.

4. Zhai L, Liu J, Zhao J, et al. Association of obesity with onset of puberty and sex hormones in Chinese girls: a 4-year longitudinal study. PLoS ONE. 2015;10(8):e0134656.

5. Wang Y. Is obesity associated with early sexual maturation? A comparison of the association in American boys versus girls. Pediatrics. 2002;110:903-910.

6. Lee J, Kaciroti N, Appugliese D, Corwyn R, Bradley R, Lumeng J. Body mass index and timing of pubertal initiation in boys. Arch Pediatr Adolesc Med. 2010;164(2):139-144.

7. Brumbaugh D, Crume T, Nadeau K, Scherzinger A, Dabelea D. Intramyocellular lipid is associated with visceral adiposity, markers of insulin resistance, and cardiovascular risk in prepubertal children: the EPOCH study. J Clin Endocrinol Metab. 2012;97(7):E1099-1105.

8. Hetemäki N, Savolainen-Peltonen H, Tikkanen MJ, et al. Estrogen metabolism in abdominal subcutaneous and visceral adipose tissue in postmenopausal women. J Clin Endocrinol Metab. 2017;102(12):4588-4595.

9. National Center for Health Statistics CfDCaP. Clinical Growth Charts. 2017; https://www.cdc.gov/growthcharts/clinical_charts. htm\#Set1.
10. Jaiswal M, Crume T, Vehik K, et al. Is low birthweight associated with adiposity in contemporary U.S. youth? J Dev Orig Health Dis. 2012;3(3):166-172.

11. Lamb M, Beers L, Reed-Gillette D, McDowell M. Feasibility of an audio computer-assisted self-interview method to self-assess sexual maturation. J Adolesc Health. 2011;48:325-330.

12. Hughes M. Analysis and design issues for studies using censored biomarker measurements with an example of viral load measurements in HIV clinical trials. Stat Med. 2000;19:3171-3191.

13. Tekindal M, Erdogan B, Yavuz Y. Evaluating left-censored data through substitution, parametric, semi-parametric, and nonparametric methods: a simulation study. Interdiscip Sci Compu Life Sci. 2017;9:153-172

14. Shoari N, Dube J. Toward improved analysis of concentration data: embracing nondetects. Environ Toxicol Chem. 2018;37:643-656.

15. Dong $\mathrm{Y}$, Peng $\mathrm{C}$. Principles missing data methods for researchers. SpringerPlus. 2013;2:222.

16. Dinse G, Jusko T, Ho L, et al. Accommodating measurements below a limit of detection: a novel application of cox regression. Am J Epidemiol. 2014;179(8):1018-1024.

17. van den Beld A, de Jong F, Grobbee D, Pols H, Lamberts S. Measures of bioavailable serum testosterone and estradiol and their relationships with muscle strength, bone density, and body composition in elderly men. J Clin Endocrinol Metab. 2000;85:3276-3282.

18. Kim C, Dabelea D, Kalyani RR, et al. Changes in visceral adiposity, subcutaneous adiposity, and sex hormones in the diabetes prevention program. J Clin Endocrinol Metab. 2017;102(9):3381-3389.

19. Mohr B, Bhasin S, Link C, O'Donnell A, McKinlay J. The effect of changes in adiposity on testosterone levels in older men: longitudinal results from the Massachusetts Male Aging Study. Eur J Endocrinol. 2006;155(3):443-452.

20. Shi Z, Araujo A, Martin S, O'Loughlin P, Wittert G. Longitudinal changes in testosterone over 5 years in community-dwelling men. $J$ Clin Endocrinol Metab. 2013;98:3289-3297.

21. Reinehr T, de Sousa G, Roth C, Andler W. Androgens before and after weight loss in obese children. J Clin Endocrinol Metab. 2005;90(10):5588-5595.

22. Reinehr T, Kulle A, Wolters B, et al. Steroid hormone profiles in prepubertal obese children before and after weight loss. J Clin Endocrinol Metab. 2013;98(6):E1022-1030.

23. Bygdell M, Kindblom J, Celind J, Nethander M, Ohlsson C. Childhood $\mathrm{BMI}$ is inversely associated with pubertal timing in normal-weight but not overweight boys. Am J Clin Nutr. 2018;108(6):1259-1263.

24. Chin VL, Williams KM, Donnelley T, et al. Long-term follow-up of gonadal dysfunction in morbidly obese adolescent boys after bariatric surgery. J Pediatr Endocrinol Metab. 2018;31(11):1191-1197.

25. Zhai L, Zhao J, Bai Y, et al. Sexual development in prepubertal obese boys: a 4-year longitudinal study. J Pediatr Endocrinol Metab. 2013;26:895-901.

26. Vissers D, Hens W, Hansen D, Taeymans J. The effect of diet or exercise on visceral adipose tissue in overweight youth. Med Sci Sports Exerc. 2016;48(7):1415-1424.

27. Stallman-Jorgensen I, Gutin B, Hatfield-Laube J, Humphries $M$, Johnson M, Barbeau P. General and visceral adiposity in black and white adolescents and their relation with reported physical activity and diet. Int J Obes (Lond). 2007;31(4):622-629.

28. Haring R, Volzke H, Felix S, et al. Prediction of metabolic syndrome by low serum testosterone levels in men. Diabetes. 2009;58:2027-2031.

29. De Simone $\mathrm{M}$, Verrotti A, lughetti L, et al. Increased visceral adipose tissue is associated with increased circulating insulin and decreased sex hormone binding globulin levels in massively obese adolescent girls. J Endocrinol Invest. 2001;24(6):438-444.

30. Moriarty-Kelsey M, Harwood J, Travers SH, Zeitler PS, Nadeau KJ. Testosterone, obesity, and insulin resistance in young males: 
evidence for an association between gonadal dysfunction and insulin resistance during puberty. J Pediatr Endocrinol Metab. 2010;23(12):1281-1287.

31. Chan JL, Matarese G, Shetty GK, et al. Differential regulation of metabolic, neuroendocrine, and immune function by leptin in humans. Proc Nat Acad Sci. 2006;103(22):8481-8486.

32. Aydin B, Winters S. Sex hormone binding globulin in children and adolescents. J Clin Res Pediatr Endocrinol. 2016;8(1):1-12.

33. Comninos A, Jayasena C, Dhillo W. The relationship between gut and adipose hormones, and reproduction. Hum Reprod Update. 2014;20(2):153-174.

34. Vandewalle S, De Schepper J, Kaufman J. Androgens and obesity in male adolescents. Curr Opin Endocrinol Diabetes Obes. 2015;22(3):230-237.
How to cite this article: Kim C, Harrall KK, Glueck DH, Shumer D, Dabelea D. Childhood adiposity and adolescent sex steroids in the Exploring Perinatal Outcomes among Children study. Clin Endocrinol (Oxf). 2019;91:525-533. https://doi. org/10.1111/cen.14058 